\title{
IMAGE RECONSTRUCTION FROM MULTIPLE SENSORS USING STEIN'S PRINCIPLE. APPLICATION TO PARALLEL MRI.
}

\author{
Alexandru Marin ${ }^{1}$, Caroline Chaux ${ }^{1}$, Jean-Christophe Pesquet ${ }^{1}$ and Philippe Ciuciu ${ }^{2}$ \\ ${ }^{1}$ Univ. Paris-Est, LIGM UMR CNRS 8049 \\ 5 Bd Descartes, 77454 Marne-la-Vallée, France \\ E-mails: alex@seneka.ro, \{caroline. chaux, jean-christophe.pesquet $\} @ u n i v-p a r i s-e s t . f r$ \\ ${ }^{2}$ CEA/DSV/I2BM/Neurospin, CEA Saclay, \\ Bat. 145, Point Courrier 156, 91191 Gif-sur-Yvette cedex, France. \\ E-mail: philippe.ciuciu@cea.fr
}

\begin{abstract}
We are interested in image reconstruction when data provided by several sensors are corrupted with a linear operator and an additive white Gaussian noise. This problem is addressed by invoking Stein's Unbiased Risk Estimate (SURE) techniques. The key advantage of SURE methods is that they do not require prior knowledge about the statistics of the unknown image, while yielding an expression of the Mean Square Error (MSE) only depending on the statistics of the observed data. Hence, they avoid the difficult problem of hyperparameter estimation related to some prior distribution, which traditionally needs to be addressed in variational or Bayesian approaches. Consequently, a SURE approach can be applied by directly parameterizing a wavelet-based estimator and finding the optimal parameters that minimize the MSE estimate in reconstruction problems. Simulations carried out on parallel Magnetic Resonance Imaging (pMRI) images show the improved performance of our method with respect to classical alternatives.
\end{abstract}

Index Terms - Reconstruction, Stein's principle, wavelets, nonlinear estimation, multiple sensors, pMRI.

\section{INTRODUCTION}

Much attention has been paid to Stein's principle [1] in the recent statistical literature in order to derive MSE estimates in statistical problems involving an additive Gaussian noise. In particular, it was successfully used in wavelet-based nonlinear denoising [2]. More recently, such an approach was extended to deconvolution problems [3, 4] and it was also employed for Poisson data denoising [5]. The key advantage of Stein's principle [6] is that it does not require prior knowledge about the statistics of the unknown image. Hence, it allows us to circumvent the difficult problem of the estimation of the hyperparameters of a prior distribution as frequently encountered in Bayesian approaches.

In this paper, we propose to further extend the scope of Steinbased approaches by addressing image reconstruction problems. More precisely, we employ Stein's principle in order to propose a wavelet-based estimator relying on Linear Expansion of Thresholds (LET) functions [7, 8]. This enables us to design efficient medical image reconstruction algorithms whose parameters are optimally computed to achieve the minimum MSE. The estimator operates in

THIS IS AN INVITED PAPER IN THE SPECIAL SESSION ON "WAVELETS AND NEUROIMAGING". the wavelet transform domain and deals with mutiple observations delivered by several sensors. The interest of this method is demonstrated in the parallel Magnetic Resonance Imaging (pMRI) context $[9,10]$. Recall that this modality offers a means of significantly reducing the acquisition time at the expense of a degraded signal-tonoise ratio. In a recent work by Chaâri et al. [11] a novel method based on a wavelet-based variational approach was proposed, which makes use of an iterative optimization algorithm to compute the Maximum A Posteriori (MAP) estimate. We aim here at proposing a faster non-iterative method where the estimator parameters are adjusted automatically. Note also that the proposed approach is very flexible concerning the wavelet choice and the estimator form.

The remainder of the paper is organized as follows. In Section 2, we briefly introduce the notation and describe the considered inverse problem. Then, Section 3 is devoted to the proposed statistical method. In particular, we show how to address the complex-valued nature of the data and build an unbiased quadratic risk estimator, before discussing the estimator choice. Finally, in Section 4, we illustrate the effectiveness of our reconstruction algorithm on a simulated set of pMRI data.

\section{PROBLEM STATEMENT}

\subsection{Notations}

We consider here pMRI images generated by considering $L$ acquisition coils and a reduction factor $R$. Let $X \times Y$ be the dimensions of the full Field Of View (FOV) image, along the respective phase and and frequency directions. The reduced FOV image size in phase encoding direction is thus given by $\Delta X=\frac{X}{R}$. $\mathbb{B}$ will denote the set of spatial indices in the reduced FOV image.

\subsection{Observation model}

At each position $\mathrm{x} \in \mathbb{B}$ we observe an $L$-dimensional data vector $\mathbf{d}(\mathbf{x})=\left[d_{1}(\mathbf{x}), \ldots, d_{L}(\mathbf{x})\right]^{\top}$, which is obtained by applying an $L \times R$ sensitivity matrix $\mathbf{S}(\mathbf{x})$ given by

$$
\mathbf{S}(\mathbf{x})=\left[\begin{array}{ccc}
S_{1}\left(x_{1}, x_{2}\right) & \ldots & S_{1}\left(x_{1}, x_{2}+(R-1) \Delta X\right) \\
\vdots & \ldots & \vdots \\
S_{L}\left(x_{1}, x_{2}\right) & \ldots & S_{L}\left(x_{1}, x_{2}+(R-1) \Delta X\right)
\end{array}\right],
$$

to an $R$-dimensional vector of pixel values in the original (unknown) image $\boldsymbol{\rho}(\mathbf{x})=\left[\rho\left(x_{1}, x_{2}\right), \rho\left(x_{1}, x_{2}+\Delta X\right), \ldots, \rho\left(x_{1}, x_{2}+(R-\right.\right.$ 
1) $\Delta X)]^{\top}$ and by adding a complex Gaussian circular noise vector corrupting samples from all coils: $\mathbf{n}(\mathbf{x})=\left[n_{1}(\mathbf{x}), \ldots, n_{L}(\mathbf{x})\right]^{\top}$. Here $\boldsymbol{\rho}(\mathbf{x})$ is an $R$-dimensional random field which is assumed to be independent of $\mathbf{n}(\mathbf{x})$. The between-coil noise covariance matrix is given by $\operatorname{Cov}\left\{\mathbf{n}(\mathbf{x}), \mathbf{n}\left(\mathbf{x}^{\prime}\right)\right\}=\mathbf{\Psi} \delta\left(\mathbf{x}-\mathbf{x}^{\prime}\right), \forall\left(\mathbf{x}, \mathbf{x}^{\prime}\right) \in \mathbb{B}^{2}$. The resulting model is consequently described by the following linear model:

$$
\mathbf{d}(\mathbf{x})=\mathbf{S}(\mathbf{x}) \boldsymbol{\rho}(\mathbf{x})+\mathbf{n}(\mathbf{x}), \quad \forall \mathbf{x} \in \mathbb{B} .
$$

The objective of this work is to recover $\boldsymbol{\rho}$ from $\mathbf{d}$ knowing $\mathbf{S}$.

\section{PROPOSED METHOD}

\subsection{Complex nature of the data}

In MRI, the acquired data are complex-valued even if the magnitude is only considered for visualization purpose. Let ${ }_{R} R$ and ${ }{ }_{I}$ be the subscripts indicating the real and imaginary parts (namely, $\Re\{\cdot\}$ and $\Im\{\cdot\}$ ) of the data. The observed data can thus be expressed by

$$
\left[\begin{array}{c}
\mathbf{d}_{\mathrm{R}}(\mathbf{x}) \\
\mathbf{d}_{\mathrm{I}}(\mathbf{x})
\end{array}\right]=\left[\begin{array}{cc}
\mathbf{S}_{\mathrm{R}}(\mathbf{x}) & -\mathbf{S}_{\mathrm{I}}(\mathbf{x}) \\
\mathbf{S}_{\mathrm{I}}(\mathbf{x}) & \mathbf{S}_{\mathrm{R}}(\mathbf{x})
\end{array}\right]\left[\begin{array}{c}
\boldsymbol{\rho}_{\mathrm{R}}(\mathbf{x}) \\
\boldsymbol{\rho}_{\mathrm{I}}(\mathbf{x})
\end{array}\right]+\left[\begin{array}{c}
\mathbf{n}_{\mathrm{R}}(\mathbf{x}) \\
\mathbf{n}_{\mathrm{I}}(\mathbf{x})
\end{array}\right]
$$

so that Model (1) can be reexpressed under the form

$$
\mathbf{d}_{C}(\mathbf{x})=\mathbf{S}_{C}(\mathbf{x}) \boldsymbol{\rho}_{C}(\mathbf{x})+\mathbf{n}_{C}(\mathbf{x}), \quad \forall \mathbf{x} \in \mathbb{B},
$$

where the involved vectors and matrices are real-valued. Assuming that we are able to compute the pseudo-inverse $\mathbf{S}_{C}^{\dagger}(\mathbf{x})$ of $\mathbf{S}_{C}(\mathbf{x})$ defined by

$$
\mathbf{S}_{C}^{\dagger}(\mathbf{x})=\left(\mathbf{S}_{C}(\mathbf{x})^{\top} \mathbf{S}_{C}(\mathbf{x})\right)^{-1} \mathbf{S}_{C}(\mathbf{x})^{\top},
$$

it follows that $\widetilde{\boldsymbol{\rho}}_{C}(\mathbf{x})=\boldsymbol{\rho}_{C}(\mathbf{x})+\widetilde{\mathbf{n}}_{C}(\mathbf{x})$ where

$$
\tilde{\boldsymbol{\rho}}_{C}(\mathbf{x})=\mathbf{S}_{C}^{\dagger}(\mathbf{x}) \mathbf{d}_{C}(\mathbf{x}) \text { and } \tilde{\mathbf{n}}_{C}(\mathbf{x})=\mathbf{S}_{C}^{\dagger}(\mathbf{x}) \mathbf{n}_{C}(\mathbf{x}) .
$$

Furthermore, the following relations regarding the second-order statistics of the random field $\widetilde{\mathbf{n}}_{C}(\mathbf{x})$ will be useful for the quadratic risk computation:

$$
\begin{aligned}
& \operatorname{Cov}\left\{\widetilde{\mathbf{n}}_{C}(\mathbf{x}), \widetilde{\mathbf{n}}_{C}(\mathbf{x})\right\}=\mathbf{S}_{C}^{\dagger}(\mathbf{x}) \mathbf{\Psi}_{C}\left(\mathbf{S}_{C}^{\dagger}(\mathbf{x})\right)^{\top} \\
& \operatorname{Cov}\left\{\widetilde{\mathbf{n}}_{C}(\mathbf{x}), \mathbf{n}_{C}(\mathbf{x})\right\}=\mathbf{S}_{C}^{\dagger}(\mathbf{x}) \Psi_{C} .
\end{aligned}
$$

where $\operatorname{Cov}\left\{\mathbf{n}_{C}(\mathbf{x}), \mathbf{n}_{C}\left(\mathbf{x}^{\prime}\right)\right\}=\Psi_{C} \delta\left(\mathbf{x}-\mathbf{x}^{\prime}\right), \forall\left(\mathbf{x}, \mathbf{x}^{\prime}\right) \in \mathbb{B}^{2}$.

\subsection{Derivation of the SURELET estimator}

For every $\mathbf{x} \in \mathbb{B}$ and $k \in\{1, \ldots, K\}$, let $\varphi_{k}(\mathbf{x}) \in \mathbb{R}^{2 L}$ be an analysis vector and let $\widetilde{\varphi}_{k}(\mathbf{x}) \in \mathbb{R}^{2 R}$ be a synthesis vector. In the case of a decomposition onto a basis of the FOV image, $K=X Y$ and the need of $2 K$ functions stems from the fact that the real and imaginary parts of the data are considered. We choose an estimator of the form

$$
\forall k \in\{1, \ldots, 2 K\}, \quad \widehat{\rho}_{k}=\theta_{k}\left(w_{k}\right)
$$

where $w_{k}=\sum_{\mathbf{x} \in \mathbb{B}} \varphi_{k}(\mathbf{x})^{\top} \mathbf{d}_{C}(\mathbf{x})$ and $\theta_{k}: \mathbb{R} \rightarrow \mathbb{R}$ is some differentiable estimating function (the choice of this function will be discussed in Section 3.4). Then the estimator of $\boldsymbol{\rho}_{C}(\mathbf{x})$ is given by

$$
\widehat{\boldsymbol{\rho}}_{C}(\mathbf{x})=\sum_{k=1}^{2 K} \widehat{\rho}_{k} \widetilde{\boldsymbol{\varphi}}_{k}(\mathbf{x})
$$

The objective now is to compute the estimator parameters that minimize the quadratic risk.

\subsection{Unbiased risk estimate}

The risk corresponding to a MSE estimation is defined as: $\forall \mathbf{x} \in \mathbb{B}$,

$$
\begin{aligned}
& \mathrm{E}\left\{\left\|\widehat{\boldsymbol{\rho}}_{C}(\mathbf{x})-\boldsymbol{\rho}_{C}(\mathbf{x})\right\|^{2}\right\} \\
& =\mathrm{E}\left\{\left\|\widehat{\boldsymbol{\rho}}_{C}(\mathbf{x})-\widetilde{\boldsymbol{\rho}}_{C}(\mathbf{x})+\widetilde{\mathbf{n}}_{C}(\mathbf{x})\right\|^{2}\right\} \\
& =\mathrm{E}\left\{\left\|\widehat{\boldsymbol{\rho}}_{C}(\mathbf{x})-\widetilde{\boldsymbol{\rho}}_{C}(\mathbf{x})\right\|^{2}\right\}+2 \mathrm{E}\left\{\widehat{\boldsymbol{\rho}}_{C}(\mathbf{x})^{\top} \widetilde{\mathbf{n}}_{C}(\mathbf{x})\right\} \\
& \quad-2 \mathrm{E}\left\{\widetilde{\boldsymbol{\rho}}_{C}(\mathbf{x})^{\top} \widetilde{\mathbf{n}}_{C}(\mathbf{x})\right\}+\mathrm{E}\left\{\left\|\widetilde{\mathbf{n}}_{C}(\mathbf{x})\right\|^{2}\right\} .
\end{aligned}
$$

Here, we focus on the second term which, by using (7), reads:

$$
\mathrm{E}\left\{\widehat{\boldsymbol{\rho}}_{C}(\mathbf{x})^{\top} \widetilde{\mathbf{n}}_{C}(\mathbf{x})\right\}=\sum_{k=1}^{2 K} \widetilde{\boldsymbol{\varphi}}_{k}(\mathbf{x})^{\top} \mathrm{E}\left\{\widehat{\rho}_{k} \widetilde{\mathbf{n}}_{C}(\mathbf{x})\right\}
$$

In addition, applying the analysis vectors $\varphi_{k}(\mathbf{x})$ to $\mathbf{d}_{C}(\mathbf{x})$ yields coefficients $w_{k}$ which can be decomposed as $w_{k}=u_{k}+n_{k}$, where

$$
\begin{aligned}
u_{k} & =\sum_{\mathbf{x} \in \mathbb{B}} \boldsymbol{\varphi}_{k}(\mathbf{x})^{\top} \mathbf{S}_{C}(\mathbf{x}) \boldsymbol{\rho}_{C}(\mathbf{x}) \\
n_{k} & =\sum_{\mathbf{x} \in \mathbb{B}} \boldsymbol{\varphi}_{k}(\mathbf{x})^{\top} \mathbf{n}_{C}(\mathbf{x}),
\end{aligned}
$$

so that $\widehat{\rho}_{k}$ can be rewritten as

$$
\widehat{\rho}_{k}=\theta_{k}\left(w_{k}\right)=\theta_{k}\left(u_{k}+n_{k}\right) .
$$

Then, by applying Stein's principle [1], it follows that:

$$
\mathrm{E}\left\{\widehat{\rho}_{k} \widetilde{\mathbf{n}}_{C}(\mathbf{x})\right\}=\mathrm{E}\left\{\theta_{k}^{\prime}\left(w_{k}\right)\right\} \mathrm{E}\left\{n_{k} \widetilde{\mathbf{n}}_{C}(\mathbf{x})\right\} .
$$

After some tedious calculations, it follows that the quadratic risk estimate reads:

$$
\begin{array}{r}
\mathrm{E}\left\{\left\|\widehat{\boldsymbol{\rho}}_{C}(\mathbf{x})-\boldsymbol{\rho}_{C}(\mathbf{x})\right\|^{2}\right\}=\mathrm{E}\left\{\left\|\widehat{\boldsymbol{\rho}}_{C}(\mathbf{x})-\widetilde{\boldsymbol{\rho}}_{C}(\mathbf{x})\right\|^{2}\right\} \\
+2 \sum_{k=1}^{2 K} \mathrm{E}\left\{\theta_{k}^{\prime}\left(w_{k}\right)\right\} \widetilde{\boldsymbol{\varphi}}_{k}(\mathbf{x})^{\top} \mathbf{S}_{C}^{\dagger}(\mathbf{x}) \boldsymbol{\Psi}_{C} \boldsymbol{\varphi}_{k}(\mathbf{x}) \\
-\operatorname{tr}\left(\mathbf{S}_{C}^{\dagger}(\mathbf{x}) \mathbf{\Psi}_{C}\left(\mathbf{S}_{C}^{\dagger}(\mathbf{x})\right)^{\top}\right) .
\end{array}
$$

An unbiased estimate of the resulting global MSE is:

$$
\widehat{\mathcal{E}}\left(\widehat{\boldsymbol{\rho}}_{C}-\boldsymbol{\rho}_{C}\right)=\widehat{\mathcal{E}}\left(\widehat{\boldsymbol{\rho}}_{C}-\widetilde{\boldsymbol{\rho}}_{C}\right)+\Delta
$$

where

$$
\begin{aligned}
& \widehat{\mathcal{E}}\left(\widehat{\boldsymbol{\rho}}_{C}-\widetilde{\boldsymbol{\rho}}_{C}\right)=\frac{1}{X Y} \sum_{\mathbf{x} \in \mathbb{B}}\left\|\widehat{\boldsymbol{\rho}}_{C}(\mathbf{x})-\widetilde{\boldsymbol{\rho}}_{C}(\mathbf{x})\right\|^{2} \\
\text { and } \quad \Delta & =\frac{2}{X Y} \sum_{k=1}^{2 K} \theta_{k}^{\prime}\left(w_{k}\right) \sum_{\mathbf{x} \in \mathbb{B}} \widetilde{\boldsymbol{\varphi}}_{k}(\mathbf{x})^{\top} \mathbf{S}_{C}^{\dagger}(\mathbf{x}) \boldsymbol{\Psi}_{C} \boldsymbol{\varphi}_{k}(\mathbf{x}) \\
& -\frac{1}{X Y} \sum_{\mathbf{x} \in \mathbb{B}} \operatorname{tr}\left(\mathbf{S}_{C}^{\dagger}(\mathbf{x}) \mathbf{\Psi}_{C}\left(\mathbf{S}_{C}^{\dagger}(\mathbf{x})\right)^{\top}\right)
\end{aligned}
$$

Note that the risk estimate is expressed in terms of observed data only. Now, the next step consists of specifying the form of the estimating functions $\theta_{k}$ in order to find the parameters that minimize the global error measure $\widehat{\mathcal{E}}(\cdot)$. 


\subsection{Estimating function choice}

Assume that the coefficients $\left(w_{k}\right)_{1 \leq k \leq 2 K}$ are classified according to $M \in \mathbb{N}^{*}$ distinct nonempty index subsets (e.g. wavelet subbands) $\mathbb{K}_{m}, m \in\{1, \ldots, M\}$. For every $m \in\{1, \ldots, M\}$ and $k \in \mathbb{K}_{m}$, we choose subband-dependent estimating functions of the form:

$$
\theta_{k}\left(w_{k}\right)=\sum_{i=1}^{I_{m}} a_{m, i} f_{m, i}\left(w_{k}\right)
$$

where $\left(a_{m, i}\right)_{1<i<I_{m}}$ are scalar real-valued weighting factors and, for every $i \in\left\{1, \ldots, I_{m}\right\}, f_{m, i}: \mathbb{R} \rightarrow \mathbb{R}$ is a differentiable function. These functions are the Linear Expension of Thresholds (LET) estimating functions introduced in $[7,8]$ and correspond to a linear combination of $I_{m} \in \mathbb{N}^{*}$ given univariate functions $f_{m, i}$ applied to $w_{k}$ (note that we use the same estimating function for a given $\mathbb{K}_{m}$ ).

\subsection{Computation of $\left(a_{m, i}\right)_{1 \leq i \leq I_{m}}$ parameters}

As mentioned earlier, we want to compute the parameters $\left(a_{m, i}\right)_{1 \leq i \leq I_{m}}$ that minimize the quadratic risk in (11). It can be shown that this amounts to solving the following set of linear equations: $\forall m \in\{1, \ldots, M\}, \forall i \in I_{m}$,

$$
\begin{aligned}
& \sum_{n=1}^{M} \sum_{j=1}^{I_{n}} a_{n, j} \sum_{\mathbf{x} \in \mathbb{B}} \beta_{m, i}(\mathbf{x})^{\top} \beta_{n, j}(\mathbf{x}) \\
& =\sum_{\mathbf{x} \in \mathbb{B}} \beta_{m, i}(\mathbf{x})^{\top} \widetilde{\boldsymbol{\rho}}_{C}(\mathbf{x}) \\
& -\sum_{k \in \mathbb{K}_{m}} f_{m, i}^{\prime}\left(w_{k}\right) \sum_{\mathbf{x} \in \mathbb{B}} \widetilde{\boldsymbol{\varphi}}_{k}(\mathbf{x})^{\top} \mathbf{S}_{C}^{\dagger}(\mathbf{x}) \Psi_{C} \boldsymbol{\varphi}_{k}(\mathbf{x})
\end{aligned}
$$

where $\beta_{m, i}(\mathbf{x})=\sum_{k \in \mathbb{K}_{m}} f_{m, i}\left(w_{k}\right) \widetilde{\boldsymbol{\varphi}}_{k}(\mathbf{x})$.

\section{SIMULATION RESULTS}

\subsection{Context}

In our experiments, the families of analysis/synthesis functions are chosen such that

$$
\begin{aligned}
& \boldsymbol{\varphi}_{k}(\mathbf{x})=\underbrace{\mathbf{S}_{C}(\mathbf{x})\left(\mathbf{S}_{C}(\mathbf{x})^{\top} \mathbf{S}_{C}(\mathbf{x})+\lambda \mathbf{I}\right)^{-1}}_{\mathbf{G}(\mathbf{x})} \psi_{k}(\mathbf{x}) \\
& \widetilde{\boldsymbol{\varphi}}_{k}(\mathbf{x})=\widetilde{\boldsymbol{\psi}}_{k}(\mathbf{x})
\end{aligned}
$$

where $\lambda>0, \mathbf{I}$ is the identity matrix and the components of vector $\boldsymbol{\psi}_{k}(\mathbf{x})$ (resp. $\widetilde{\boldsymbol{\psi}}_{k}(\mathbf{x})$ ) are obtained from shifted versions of a $2 \mathrm{D}$ separable wavelet analysis function (resp. synthesis function). A scheme summarizing the proposed reconstruction approach is given in Fig. 1.

Concerning the estimating functions, we choose $I_{m}=2 . f_{m, 1}$ is the identity function and

$$
\forall \rho \in \mathbb{R}, \quad f_{m, 2}(\rho)=\left(1-\exp \left(-\frac{\rho^{8}}{\left(\omega \sigma_{m}\right)^{8}}\right)\right) \rho
$$

where $\omega \in] 0, \infty\left[\right.$ and $\sigma_{m}$ is the standard deviation of $\left(n_{k}\right)_{k \in \mathbb{K}_{m}}$. These LET estimating functions were introduced in [7].

We compare our results with SENSE reconstruction [9], which is based on a weighted least-squares estimator:

$$
\widehat{\boldsymbol{\rho}}_{\mathrm{SENSE}}(\mathbf{x})=\left(\left(\mathbf{S}^{*}(\mathbf{x})\right)^{\top} \boldsymbol{\Psi}^{-1} \mathbf{S}(\mathbf{x})\right)^{\dagger}\left(\mathbf{S}^{*}(\mathbf{x})\right)^{\top} \boldsymbol{\Psi}^{-1} \mathbf{d}(\mathbf{x}) .
$$

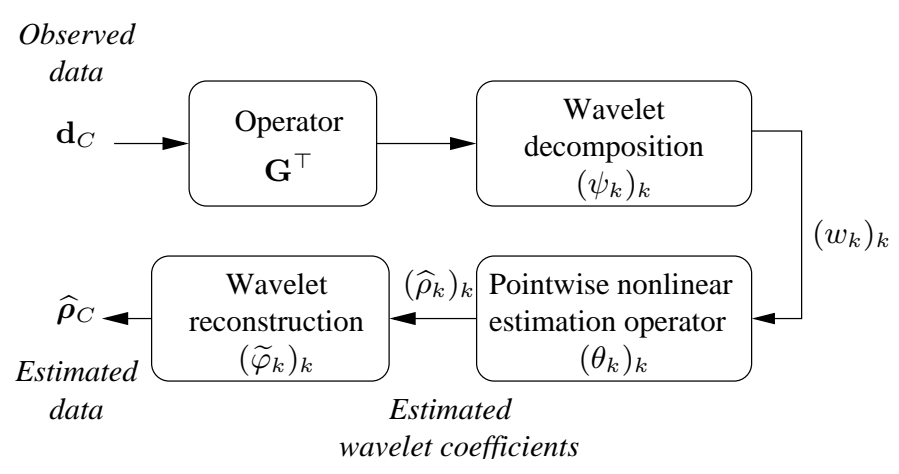

Fig. 1. Flowchart of the proposed reconstruction method.

\subsection{Numerical results}

To assess the performance of the proposed method, we simulated synthetic data from a full FOV reference image displayed in Fig. 2(a) according to model (1). Note that the dynamic range of the absolute value of the pixels is large.

The number of antennas is here equal to $L=8$ and the reduction factor is $R=4$. The covariance matrix of the noise $\psi$ is chosen to be diagonal (no between coil correlation) and all the diagonal entries are chosen equal to $\sigma^{2}$ (homoscedasticity). Next, we compared our results with those provided by the classical SENSE reconstruction. Here, an orthonormal wavelet analysis (symlets of length 8)

\begin{tabular}{|c|c|c|c|c|}
\hline \multicolumn{2}{|c|}{ Method } & Real Part & Imag. Part & Magn. \\
\hline & & \multicolumn{3}{|c|}{$\sigma^{2}=510^{6}$} \\
\hline \multicolumn{2}{|c|}{$\overline{\text { SENSE }}$} & 36.29 & 35.48 & 38.75 \\
\hline \multirow{3}{*}{ Proposed } & $\lambda=10^{-3}$ & 38.28 & 36.77 & 40.43 \\
\hline & $\lambda=10^{-4}$ & 38.30 & 36.79 & 40.46 \\
\hline & $\lambda=10^{-5}$ & 38.30 & 36.79 & 40.46 \\
\hline & & \multicolumn{3}{|c|}{$\sigma^{2}=12.510^{5}$} \\
\hline \multicolumn{2}{|c|}{$\overline{\text { SENSE }}$} & $\overline{42.94}$ & $\overline{42.40}$ & $\overline{45.58}$ \\
\hline Proposed & $\lambda=10^{-4}$ & 43.57 & 42.74 & 46.35 \\
\hline & & \multicolumn{3}{|c|}{$\sigma^{2}=210^{7}$} \\
\hline \multicolumn{2}{|c|}{$\overline{\text { SENSE }}$} & $\overline{\overline{30.00}}$ & $\overline{28.57}$ & $\overline{32.03}$ \\
\hline Proposed & $\lambda=10^{-4}$ & 33.72 & 31.69 & 34.92 \\
\hline
\end{tabular}
is performed on 4 resolution levels. The regularization parameter $\lambda$ was chosen empirically. Typical values around 0.001 were observed to provide accurate and reliable results. The performance was measured in terms of Peak Signal-to-Noise Ratio (PSNR) as reported in Table 1. In short, our method enables a significant gain in PSNR values with respect to the SENSE approach.

Table 1. PSNR values for reconstructed images (in dB).

Interestingly, the larger the noise, the stronger the PSNR improvements we observed in favour of the proposed approach. For visualization purposes, the reconstructed images by SENSE and SteinLet methods are shown in Fig. 2(b)-(c) when $\sigma^{2}=510^{6}$. The main differences can be observed in the center of the images where more residual noise is found for SENSE reconstruction. Note that the Stein-LET reconstruction in Fig. 2(c) is achieved in about $15 \mathrm{~s}$. on a Xeon(R) CPU X5450@3.00GHz. 
(a)

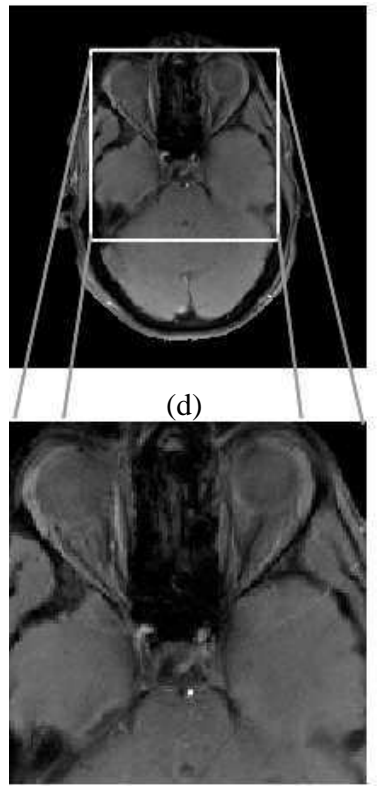

(b)

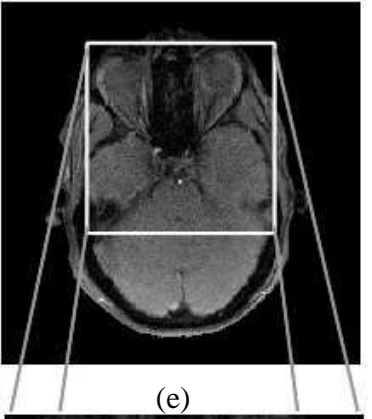

(c)

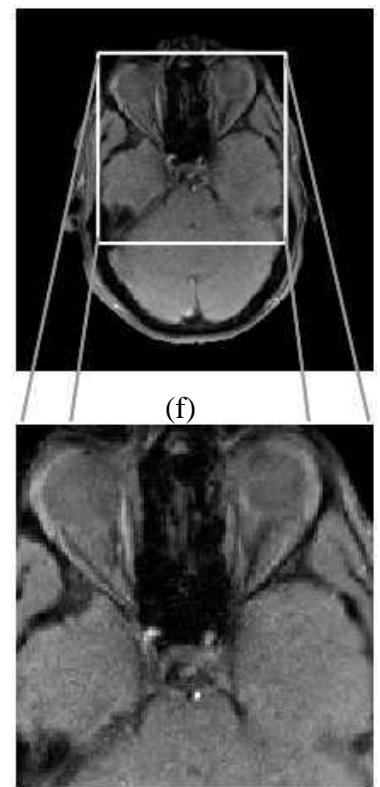

Fig. 2. (a): Magnitude of the reference image: range in [0,91496]. (b)-(c): Magnitude of the restored images by SENSE and the proposed Stein-LET approach, respectively. (d)-(f): zoomed versions of images (a)-(c) on the central part of the brain to illustrate the noise reduction using the Stein-LET estimator.

\section{CONCLUSION}

We proposed a new reconstruction method based on Stein's principle and LET functions which was applied to pMRI. In this context, our approach provides more accurate results than the classical SENSE alternative. In our approach, most of the parameters are set in an unsupervised manner. In addition, the computational complexity is quite reasonable. In the future, we plan to validate this restoration method in a more realistic context i.e. without knowing the amount of noise and using an unperfectly known sensitivity matrix. Furthermore, we will consider 3D decomposition to extend the present contribution and enable the reconstruction of all slices simultaneously. It would be also interesting to apply this approach to other reconstruction strategies $[12,13]$.

\section{REFERENCES}

[1] C. Stein, "Estimation of the mean of a multivariate normal distribution," Ann. Stat., vol. 9, no. 6, pp. 1135-1151, Nov. 1981.

[2] D. L. Donoho and I. M. Johnstone, "Adapting to unknown smoothness via wavelet shrinkage," J. American Statist. Ass., vol. 90, pp. 1200-1224, Dec. 1995.

[3] J.-C. Pesquet, A. Benazza-Benyahia, and C. Chaux, "A SURE approach for digital signal/image deconvolution problems," IEEE Trans. Signal Process., vol. 57, no. 12, pp. 4616-4632, Dec. 2009.

[4] C. Chesneau, M.J. Fadili, and J.-L. Starck, "Stein block thresholding for wavelet-based image deconvolution," Electronic Journal of Statistics, vol. 4, pp. 415-435, 2010.

[5] F. Luisier, T. Blu, and M. Unser, "Undecimated Haar thresholding for Poisson intensity estimation," in Proc. of the 2010
IEEE Int. Conf. on Image Processing (ICIP'10), Hong Kong, People's Republic of China, Sep. 26-29, 2010, pp. 1697-1700.

[6] M. Raphan and E. P. Simoncelli, "Learning least squares estimators without assumed priors or supervision," Tech. Rep. Computer Science TR2009-923, Courant Inst. of Mathematical Sciences, New York University, Aug. 2009.

[7] T. Blu and F. Luisier, "The SURE-LET approach to image denoising," IEEE Trans. Image Process., vol. 16, no. 11, pp. 2778-2786, Nov. 2007.

[8] J.-C. Pesquet and D. Leporini, "A new wavelet estimator for image denoising," in IEE Sixth Int. Conf. Im. Proc. Appl., Dublin, Ireland, Jul. 14-17 1997, vol. 1, pp. 249-253.

[9] K. P. Pruessmann, M. Weiger, M. B. Scheidegger, and P. Boesiger, "SENSE: sensitivity encoding for fast MRI," Magnetic Resonance in Medicine, vol. 42, no. 5, pp. 952-962, Jul. 1999.

[10] C. Rabrait, P. Ciuciu, A. Ribès, C. Poupon, P. Leroux, V. Lebon, G. Dehaene-Lambertz, D. Le Bihan, and F. Lethimonnier, "High temporal resolution functional MRI using parallel echo volume imaging," Magnetic Resonance Imaging, vol. 27, no. 4, pp. 744-753, Mar. 2008.

[11] L. Chaâri, J.-C. Pesquet, A. Benazza-Benyahia, and P. Ciuciu, "A wavelet-based regularized reconstruction algorithm for SENSE parallel MRI with applications to neuroimaging," Medical Image Analysis, 2011, In press, doi:10.1016/j.media.2010.08.001.

[12] J. Petr, J. Kybic, M.1 Bock, S. Müller, and V. Hlaváč "Parallel image reconstruction using B-spline approximation (PROBER)," Magn. Reson. Med., vol. 58, no. 3, pp. 582-591, Sep. 2007.

[13] A. A. Samsonov, "On optimality of parallel MRI reconstruction in k-space," Magn. Reson. Med., vol. 59, no. 1, pp. 156164, Jan. 2008. 